\title{
Optimization of the Backpropagation Method with Nguyen-widrow in Face Image Classification
}

DOI: https://doi.org/10.47175/rissj.v2i2.226

\author{
| Ichsanuddin Hakim ${ }^{1, *} \mid$ Syahril Efendi ${ }^{2}$ | Pahala Sirait ${ }^{3}$ |
}

\author{
1, 2, 3 Department of Computer \\ Science and Information \\ Technology, University of \\ North Sumatra, Indonesia
}

\begin{abstract}
In this resarch, the Backpropagation method can be optimized using the Nguyen-Widrow algorithm which is related to the initialization of weights and bias. With the optimization of the Backpropagation method using the Nguyen-Widrow algorithm, facial images can be recognized more quickly and accurately. In the trial process carried out on hidden layer 6 neurons with a target error of 0.01, by default the Backpropagation method produces an accuracy rate of $96 \%$, whereas if the Backpropagation method is optimized it will produce a higher accuracy rate of $98 \%$. Similarly, if it is done on hidden layers 7, 8, 9 and 10 neurons. From the results of this research, these algorithms and methods can be used to improve information technology and play a role in facilitating all aspects of human activities. With the speed and high level of accuracy in taking pictures of faces, it can be used as a policy or provision that is applied to traffic cameras, cameras at train stations to airports and surveillance cameras placed in other strategic locations and can help in the social life and human security.

KEYWORDS

Artificial Neural Network; Backpropagation; Nguyen-widrow; image processing; face image; classification
\end{abstract}

\section{INTRODUCTION}

Image is a two-dimensional function with the symbols $f(x, y), x$ and $y$ are spatial coordinates, and $f$ the amplitude of each pair of coordinates $(x, y)$, usually called grayscale (Wayahdi $\&$ Lydia, 2020). In fact, in many cases $f(x, y)$ which we might think of as a physical signal hitting a 2D sensor face is actually a function of many variables including depth $(z)$, color $(\lambda)$, and time $(t)$ (Young \& Vliet, 2007). The image classification process is a field of artificial intelligence that is widely used for scientific purposes such as pattern recognition, face recognition, decision making, prediction, and others.

Artificial Intelligence (AI) is a cutting-edge science for developing and studying several theories, methods, technical, and applied systems to simulate and expand human intelligence (Ren \& Wang, 2019). At present, almost all computer features along with the latest electronics practice artificial intelligence (Budiharto \& Suhartono, 2014). Many artificial intelligence methods can be used in image classification, including Backpropagation neural networks. Artificial Neural Network (ANN) is a mathematical discovery inspired by observations made during the study of biological systems (Fikri, Cheddad, \& Sabri, 2019). The ANN learning algorithm allows approximate input-output mapping of multivariate and non-linear functions (Demby's, Gao, \&Desouza , 2019)

The Backpropagation method is a method inspired by the human brain, where neurons are non-linearly connected to each other, which is a reliable Artificial Neural Network in case of problem solving (Wayahdi, Zarlis , \& Putra, 2019). Backpropagation is a method that uses supervised learning procedures. This algorithm takes advantage of signal errors, 
the neural network modifies the weight of its synaptic connections to improve system performance (Kharola \& Kumar, 2014). The purpose of back propagation is to modify the weights to train the neural network to correctly map inputs to outputs (Amrutha \&Remya, 2018).

Neupane \& Shakya in their research detecting network intrusion using the Backpropagation method explained that neural networks can characterize examples, the results of which can be used as part of an intrusion detection system for attack classification. Back propagation calculations are well used for the preparation of neural networks as a promising element (Neupane \& Shakya, 2017). Meanwhile, Kharola\& Kumar explained that the Backpropagation method is a method that can learn very quickly and produce more accurate future weather predictions (Kharola \& Kumar, 2014).

The Backpropagation method requires initializing the initial weights in the training and testing process. Usually, the initialization weights are determined randomly. This results in less efficient performance of the Backpropagation method because the training time can be very long, therefore the algorithm proposed in the weight initialization in this study is Nguyen-widrow. Nguyen-widrow is a neural network weight initialization algorithm to reduce training time. In the initialization of weights, a small number of random values for the weights are assigned to the operation of the Backpropagation network (Wayahdi, Zarlis, \& Putra, 2019).

Wayahdi et al. in his research on the classification of temperature data, using the Nguyenwidrow algorithm, on the Backpropagation method to initialize the weights and get the results of accelerating the training time about twice as fast without reducing the level of accuracy (Wayahdi, Zarlis, \& Putra, 2019). Meanwhile, Masood et al. explained that in order to get the best results in the case obtained from experimental statistical analysis, it is highly recommended to use the Nguyen-widrow algorithm to initialize the weights resulting in a minimum test error value (Masood, Doja, \& Chandra, 2016).

From the above explanations, the neural network Backpropagation method will be optimized with the Nguyen-widrow algorithm as an initialization weight. The method used will be further analyzed to improve accuracy and reduce squared error in the face image classification process. Based on the accuracy of the facial image classification process, it can help improve the accuracy in monitoring human faces and detecting data quickly. This research will also have an impact on advances in information technology, especially in the field of security systems on computer networks to anticipate disturbances or threats of resource theft, it is necessary to prevent and control network security systems (Nasution, A.M, et.al. 2021). This facial recognition system can be applied in the form of monitoring so that it can detect and catch suspected people. The system can be applied in agencies that are connected to large to medium scale networks such as train stations, airports, hospitals, traffic to schools. So that it can be useful in human social life.

\section{RESEARCH METHODS}

At this stage, an analysis of image extraction is carried out to obtain knowledge about the algorithm and method being analyzed, namely the Nguyen-widrow algorithm as a weight initialization of the Backpropagation neural network method. The process flow of this research, namely:

\section{Image Processing}

Image processing begins by taking a sample of 150 images of the face. The image obtained is then selected to be the main part of the face, then resized to $100 \times 100$ pixels and cut into several parts such as the eyes, nose, and lips (mouth). After that the image is extracted to produce the mean value for the input variable value $\left(x_{i}\right)$ and target $(y)$ as a 
dataset. Illustration of image processing starting from taking pictures to becoming a dataset can be seen in the picture.

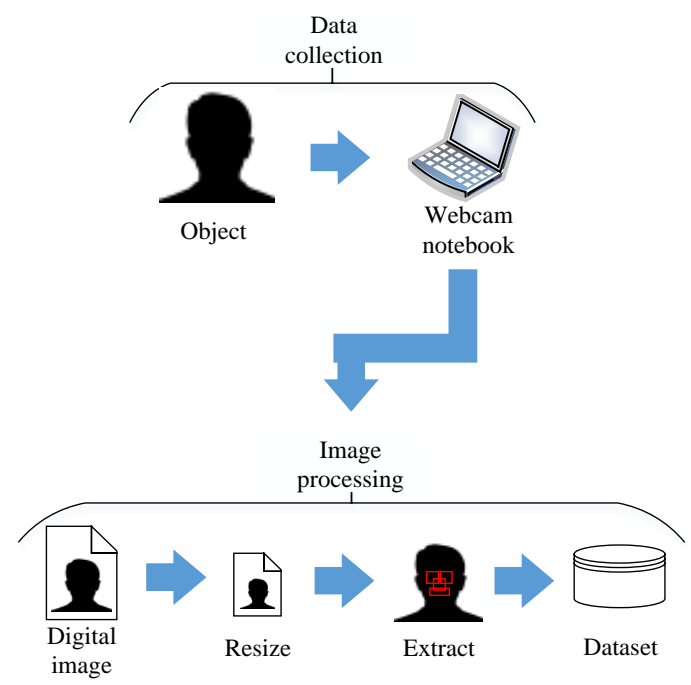

Figure 1. Image Processing

\section{Data Cleaning \& Preparation}

There is a term Garbage In - Garbage Out which means that the results of the model building will be bad if the input is also bad. The data obtained still has many shortcomings, so it needs to be processed (cleaning) first. General things that can be done at the data cleaning stage include format consistency, data scale, data duplication, missing value, and skewness. Data preparation is needed as initial preparation when cleaning data has been completed.

\section{Data Storage}

Processed data is entered into certain data stores so that it can be processed again at a later time. The tools used to support this are the concept of the Relational Database Management System (RDBMS).

\section{Data Classification}

Before processing the classification, the dataset is normalized first so that the distance between one value and another is not significantly different. The normalization process is carried out using the formula:

$$
x^{\prime}=\frac{0.8(x-a)}{b-a}+0.1
$$

Where $a$ is the minimum value of the dataset, $b$ is the maximum value of the dataset, $x$ is the data to be normalized, while $x$ 'is the result of normalization.

Then initial initialization will be given such as: number of hidden layer neurons, initial weight of input to hidden layer, initial weight of bias to hidden layer, initial weight of hidden layer to output layer, initial weight of bias to output layer, learning rate, maximum epoch, and target error randomly for the classification process with Backpropagation. Then put the Nguyen-widrow algorithm as the initialization weight as an effort to optimize the Backpropagation method in the image classification process. In this study, the dataset is divided into training data and testing data. The description of the Artificial Neural Network model in this study can be seen in Figure 2. 


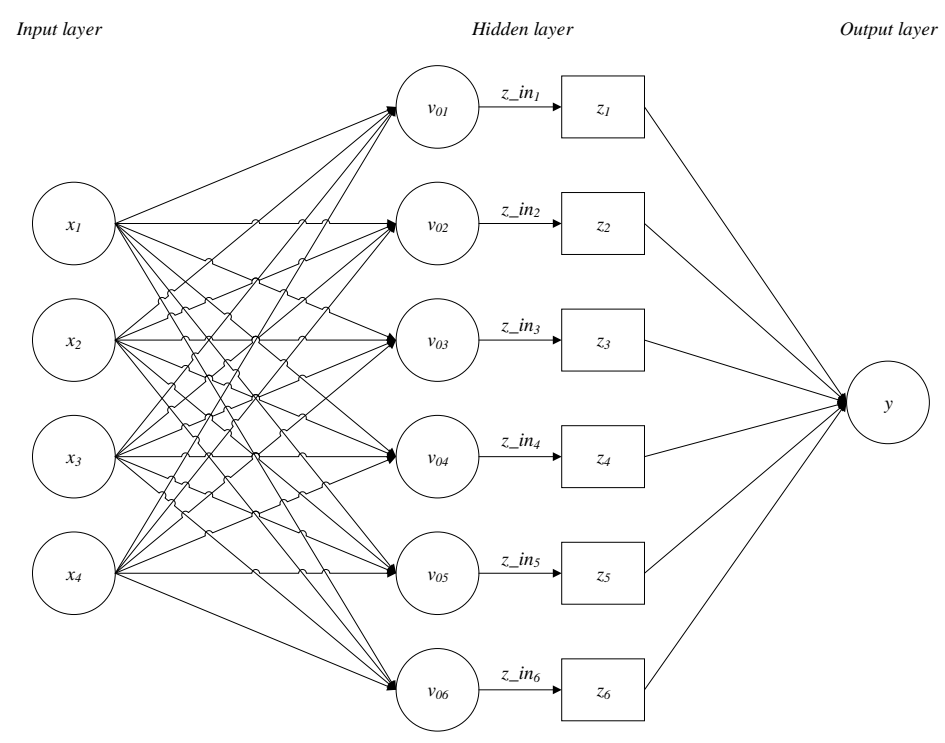

Figure 2. Artificial Neural Network Model

In figure 2, it shows that the Artificial Neural Network model is built with input layer 4 neurons, hidden layer 6 neurons, and output layer 1 neurons. The Artificial Neural Network model that is built can be modified or varied the number of neurons in the hidden layer. Testing will be carried out with variations in the number of neurons in the hidden layer to obtain the best model.

\section{Analysis}

After the classification process is carried out, the next stage is the analysis process in which the results of the image classification using the Backpropagation method with random weights are compared with the results of the Backpropagation method image classification with initialized weights using the Nguyen-widrow algorithm. At this stage it can be seen whether the initialization of weights with Nguyen-widrow can speed up the training process and improve accuracy and can reduce the value of the squared error or not.

\section{RESULTS AND DISCUSSION}

This section describes the results of research and discussion of face classification that can be done by optimizing the Backpropagation neural network method with the Nguyenwidrow algorithm. The analysis process begins by determining the existing training image in the dataset. Then the dataset is trained according to initial initialization values such as weight and bias values. Repeat training as many epochs as specified or target error specified. Save the new weights and bias values for testing.

In this test using 10 test data with the number of neurons in the varied hidden layer, namely 6 neurons, 7 neurons, 8 neurons, 9 neurons, and 10 neurons. As well as variations of the target error ranging from $0.05,0.04,0.03,0.02$, and 0.01 . This applies to the initialization of random weights as well as with Nguyen-widrow. The total test data were all tested 500 times.The values used in the input layer are eyes and eyebrows (right and left), nose, and lips (mouth) by providing the initials $x_{1}, x_{2}, x_{3}$, and $x_{4}$. While the output layer or the target in the test is given the initials $y$. Result of training data with learning rate $=1$, max. epoch $=$ 10,000 , target error $=0.05$ to 0.01 , with hidden layer $=6$ neurons to 10 neurons can be seen in Table 1. 
Table 1. Results of Training Data

\begin{tabular}{|c|l|c|c|c|c|c|}
\hline \multirow{2}{*}{$\begin{array}{c}\text { Hidden } \\
\text { layer }\end{array}$} & \multirow{2}{*}{ Method } & \multicolumn{5}{|c|}{ Target error \& Epoch } \\
\cline { 2 - 7 } & & $\mathbf{0 . 0 5}$ & $\mathbf{0 . 0 4}$ & $\mathbf{0 . 0 3}$ & $\mathbf{0 . 0 2}$ & $\mathbf{0 . 0 1}$ \\
\hline \multirow{2}{*}{6} & $\mathrm{~B}$ & 350 & 587 & 819 & 1,021 & 2,213 \\
\cline { 2 - 7 } & $\mathrm{B}+\mathrm{N}$ & 288 & 318 & 544 & 739 & 909 \\
\hline \multirow{2}{*}{8} & $\mathrm{~B}$ & 451 & 631 & 901 & 1,119 & 2,241 \\
\cline { 2 - 7 } & $\mathrm{B}+\mathrm{N}$ & 288 & 466 & 638 & 835 & 967 \\
\hline \multirow{2}{*}{9} & $\mathrm{~B}$ & 601 & 708 & 892 & 1,012 & 2,184 \\
\cline { 2 - 7 } & $\mathrm{B}+\mathrm{N}$ & 437 & 533 & 618 & 842 & 986 \\
\hline \multirow{2}{*}{10} & $\mathrm{~B}$ & 790 & 880 & 973 & 1,291 & 2,231 \\
\cline { 2 - 7 } & $\mathrm{B}+\mathrm{N}$ & 528 & 605 & 701 & 912 & 997 \\
\cline { 2 - 7 } & $\mathrm{B}$ & 932 & 1,017 & 1,878 & 1,902 & 2,203 \\
\hline
\end{tabular}

$\mathrm{B}=$ Backpropagation

$\mathrm{B}+\mathrm{N}=$ Backpropagation dan Nguyen-widrow

Table 1 shows the results of the test on the image dataset by comparing the results of the epoch from the Backpropagation method and Backpropagation optimization with Nguyenwidrow on hidden layers 6 to 10 neurons. The training resulted that the smaller the target error, the greater the number of epochs. It can also be seen that the number of epochs using the Backpropagation and Nguyen-widrow methods produces a smaller number of epochs compared to training using only the standard Backpropagation method. After the training process is complete, the next step is to save the weight of the training results. The training result weights will be used as the initial weights in the testing process. The comparison of the number of epochs in the training process can be visualized in graphical form in Figure 3.

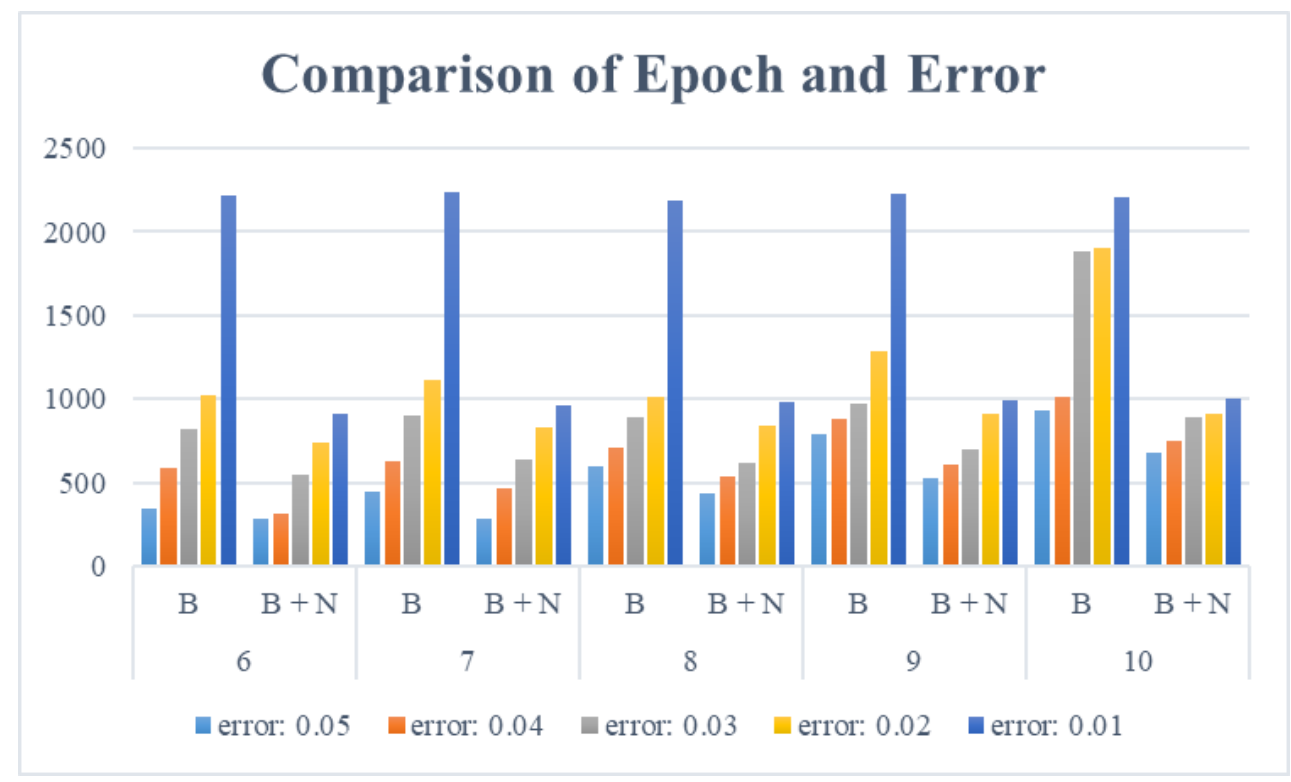

Figure 3. Visualization of Comparison of Epoch and Error

In Figure 3 it can be seen the comparison of the number of epochs and errors of the standard Backpropagation method and the optimization of the Backpropagation method with the Nguyen-widrow algorithm. The number of epochs obtained by the optimization method is smaller than the standard method.The test results with hidden layer 6 neurons to hidden layer 10 neurons can be seen in Figure 4. 


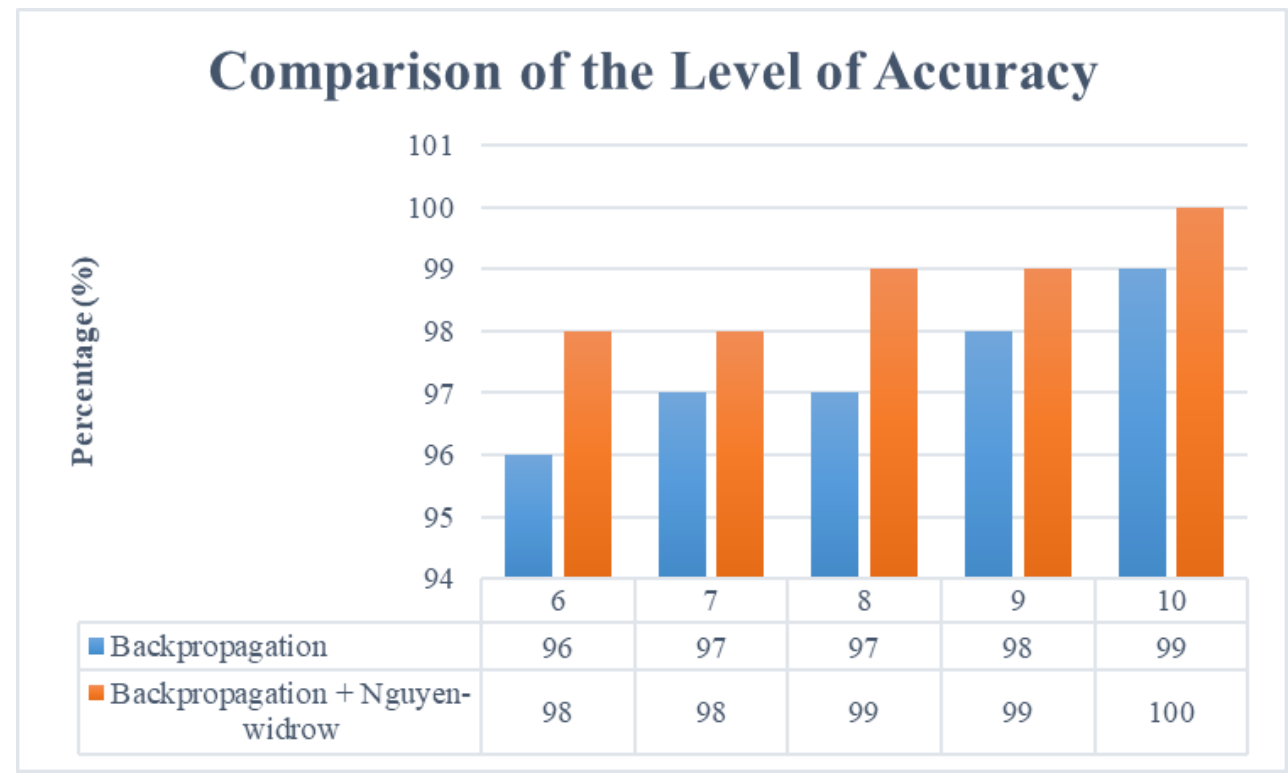

Figure 4. Visualization of Comparison of the Level of Accuracy

In Figure 4 it can be seen a comparison of the accuracy level of the test results of the standard Backpropagation method with Backpropagation optimization (with Nguyenwidrow). Backpropagation with Nguyen-widrow results in an accuracy rate higher than 500 times the test performed. It can be seen in the hidden layer 10 neurons, the Backpropagation method with Nguyen-widrow can obtain an accuracy of 100\%. This shows that the Nguyenwidrow algorithm is highly recommended in the weight initialization of the Backpropagation neural network method.

\section{CONCLUSION}

Based on the results of testing and analysis for classifying facial images using the Backpropagation method with the Nguyen-widrow algorithm method, it proves that this method recognizes facial patterns quite well. In terms of weight and bias, the Backpropagation method can be optimized using the Nguyen-Widrow algorithm, so that it can find out facial images more quickly and accurately.

By default the Backpropagation method can recognize training patterns at epoch 2.213 on hidden layer 6 neurons with a target error of 0.01. Meanwhile, if the Backpropagation method is optimized, it can recognize training patterns at the 909th epoch. When the testing process is carried out on hidden layer 6 neurons with a target error of 0.01 , the Backprogation method defaults to $96 \%$ accuracy, while optimization in the Backpropation method has a high accuracy that is $98 \%$. Likewise, if the hidden layers 7, 8, 9 and 10 neurons are optimized for the Backpropagation method, the test results will recognize training patterns faster with higher accuracy.

So, with $98 \%$ accuracy it is very high quality to capture and monitor facial images with small errors to use as a security camera to help humans. Such as security cameras in traffic protection, CCTV, and others.

\section{REFERENCES}

Amrutha, J. \& Remya, A.A.S. (2018). Performance Analysis of Backpropagation Algorithm of Artificial Neural Networks in Verilog. IEEE 3rd International Conference on Recent Trends in Electronics, Information, \& Communication Technology (RTEICT), pp 1547-1550. 
Budiharto, W. \&Suhartono, D. (2014). Artificial Intelligence. Andi (Handbook). ISBN: 978979-29-4222-4.

Demby's, J., Gao, Y., \& Desouza, G.N. (2019). A Study on Solving the Inverse Kinematics of Serial Robots using Artificial Neural Network and Fuzzy Neural Network. IEEE International Journal, pp 1-6.

Fikri, M., Cheddadi, B., \&Sabri, O. (2019). Predicting Moroccan Real Network's Power Flow Employing the Artificial Neural Network. IEEE International Journal, pp 1-6.

Kharola, M. \& Kumar, D. (2014). Efficient Weather Prediction by Back-propagation Algorithm. IOSR Journal of Computer Engineering (IOSR-JCE). e-ISSN: 2278-0661, pISSN: 2278-8272, Vol. 16, Issue 3, Ver. IV, pp 55-58.

Masood, S., Doja, M.N., \& Chandra, P. (2016). Analysis of Weight Initialization for Scaled Conjugate Gradient Training Algorithm. IEEE 2nd International Conference on Computational Intelligence \& Communication Technology, pp 533-538.

Nasution, A.M., Zarlis, M., Suherman. (2021). Analysis and Implementation of Honeyd as a Low-Interaction Honeypot in Enhancing Security Systems. Randwick International of Social Sciences Journal, 2 (1), 124-135, DOI: https://doi.org/10.47175/rissj.v2i1.209

Neupane, N. \& Shakya, S. (2017). Comparative Analysis of Backpropagation Algorithm Variants for Network Instrusion Detection. IEEE International Conference on Computing, Communication, and Automation (ICCCA), pp 726-729.

Ren, Z., Xu, Z., \& Wang, H. (2019). The Strategy Selection Problem on Artificial Intelligence with An Integrated VIKOR and AHP Method under Probabilistic Dual Hesitant Fuzzy Information. IEEE International Journal, pp 1-23.

Wayahdi, M.R., Zarlis, M., \& Putra, P.H. (2019). Initialization of the Nguyen-widrow and Kohonen Algorithm on the Backpropagation Method in the Classifying Process of Temperature Data in Medan. IOP Conference Series: Journal of Physics the 3rd International Conference on Computing and Applied Informatics, pp 1-6.

Wayahdi, M.R., Tulus, \& Lydia, M.S. (2020). Combination of k-Means with Nä̈ve Bayes Classifier in the Process of Image Classification. IOP Conference Series: Materials Science and Engineering 3rd NICTE, pp 1-6.

Young, I.T., Gerbrands, J.J., \& Vliet, L.J. (2007). Fundamentals of Image Processing. Delft University of Technology (handbook), pp 1-112. 